American Journal of Clinical Research and Reviews
(ISSN:2576-0505)

\title{
Clinical and Histopathological Findings in Patients with Follicular Dermatoses: All Skin Diseases Starts in the Hair Follicles as New
}

\section{Hypothesis}

\section{${ }^{*}$ Professor Khalifa E. Sharquie MD,PhD,FRCP Edin, *Professor Adil A. Noaimi MD, DDV, FICMS, ${ }^{* *}$ Dr. Rusul A. Flayih MD.}

*Department of Dermatology, College of Medicine, University of Baghdad. Iraqi and Arab Board for Dermatology and Venereology, Baghdad Teaching Hospital, Medical City, Baghdad, Iraq.

** Center of Dermatology; Baghdad Teaching Hospital; Medical City, Baghdad, Iraq.

\section{ABSTRACT}

Background: Follicular dermatoses are group of skin diseases characterised by follicular lesions during the course of the disease. Objective: To gather all skin diseases where there is follicular lesions to be evaluated in order to reach the hypothesis that hair follicles are the most princi-ple target area in the etiopathogenesis of these skin disorders. Patients and Methods: This is a descriptive, clinical and histopathological study that was carried out at the Department of Dermatology, Baghdad Teaching Hos-pital, Medical City, and Baghdad, Iraq during the period from November 2018 to August 2019. Sixty patients with different follicular dermatoses were enrolled and all socio- demographic data related to the disease was obtained from each patient, in addition to physical examination was performed for each patient. Bi-opsies were taken from 40 patients, for histopathological evaluation. Results: Sixty patient, were included in this study, 35 patients males and 25 pa-tients females with male to female ratio 1.4:1. Their ages ranged from 1-80(30.2 \pm 16.96 ) years. All patients had follicular lesions. The histopathological results showed: perifollicular lymphocytes, follicular invasion and degeneration, follicu-lar plugging, fibrous tract remnant, perifollicular melanophages and absent mel-anin pigment in the epidermis of patients with vitiligo. Conclusion: The result of the present study showed that all skin diseases start in the hair follicles and might spread to involve the proper epidermis, and the le-sions could be microscopic or visible to the naked eye. The hair follicles have many anatomical and immunological privileges that make it easy to be an early target for any cutaneous pathological process. Hence these findings are in sup-port of new hypothesis that say: all skin diseases starts in the hair follicles and then spread to attack the adjacent proper epidermis

Keywords: follicular dermatoses, hair follicle, immunological privilege
${ }^{*}$ Correspondence to Author:

Professor Khalifa E. Sharquie, Department of Dermatology, College of Medicine, University of Baghdad. Iraqi and Arab Board for Dermatology and Venereology, Baghdad Teaching Hospital, Medical City, Medical Collection Office, P.O. Box 61080 Postal Code 12114,Baghdad, Iraq.

How to cite this article:

Khalifa E. Sharquie, Adil A. Noaimi, Rusul A. Flayih. Clinical and Histopathological Findings in Patients with Follicular Dermatoses: All Skin Diseases Starts in the Hair Follicles as New Hypothesis. American Journal of Clinical Research and Reviews, 2020; 4:17.

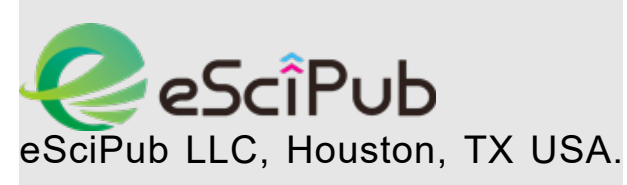

Website: https://escipub.com/ 


\section{Introduction}

There are many follicular dermatoses where the hair follicles are involved during the course of the disease but what have been published in medical literature are not many like acne vulgaris, atopic dermatitis, keratosis pilaris, discoid lupus erythematosus and follicualr lichen planus. 1-3 But, during prolonged daily clinical practice we have noticed that many other dermatoses that have follicular lesions during the course of the disease. These lesions might be seen by naked eye as follicular papules, pustules, nodules and plaques or if not seen, either these follicular lesions gone or could be microscopic and could not be detected. This observation has encouraged us to ask why these follicular lesions are commonly detected in clinical practice. The answer is that hair follicles have very distinctive anatomical site and structure as they are suspended inside the dermis where they are easily exposed to many pathological factors. In addition hair follicles have immunological privileges that make them away from many immunological agents. For all these reasons make hair follicles to be easily exposed to more marked inflammatory reactions when compared with neighbouring epidermis, ${ }^{4}$, thus triggering many skin diseases like vitiligo, psoriasis, seborrhoic dermatitis, and numerous other skin diseases ${ }^{6-8}$.

So the objective of this work is to evaluate skin diseases and to do full clinical and histopathological examination in order to support the hypothesis that says: all skin diseases start primarily and initially in the hair follicles.

\section{Patients and Methods}

This is a descriptive clinical and histopathological study that was carried out at the Department of Dermatology, Baghdad Teaching Hospital; Medical City-Baghdad,-Iraq during the period from November 2018 to August 2019.

Formal consent was given from each patient and/or their parents after full explanation of the nature of this study. The ethical approval was granted from the Scientific Committee of the Scientific Council of Dermatology, Iraqi Board for Medical Specializations. Sixty patients with clinically follicular diseases were enrolled. The demographic information like age, gender, occupation, marital status, age of the disease at presentation, associated symptoms, onset, family history of the same disease, past medical, surgical and drug history were recorded. The clinical examination was done in each patient regarding sites involved, morphology, and any associated features. Medical photographic documentation of the lesional at variable sites was done by using Samsung Galaxy ${ }^{\circledR}$ S6 12megapixel digital camera in the same place and illumination. Skin biopsy was carried out from forty patients, four milometers in size, was taken from the most representative lesional sites. The specimens then saved in the formalin and sent to the laboratory, to be submitted for vertical sectioning and Haematoxylin-Eosin staining. Data were statistically described in term of range, mean, standard deviation, frequencies and relative frequencies by using SPSS $\circledast$ Software.

\section{Results}

Sixty patient, were included in this study, 35 patients males and 25 patients females with male to female ratio $1.4: 1$. Their ages ranged from 1-80 years with a mean \pm standard deviation (SD) of the age was $30.2 \pm 16.96$ years. The lesional biopsy were taken from forty patients $23(57.5 \%)$ male and $17(42.5 \%)$ female, while the remaining twenty patient were diagnosed clinically $12(60 \%)$ males and $8(40 \%)$ females. The socio-demographic features were shown in (Table-1).

Clinical results: all patients that were examined clinically, showed follicular involvement in different body sites in a form of macules, papules, pustules, nodules and plaques. The associated signs in the form of scales, erythema, crustation, patchy hair loss, atrophy, scarring, and dyspigmentation of skin were documented. The clinical diagnosis of diseases was including psoriasis, vitiligo, atopic dermatitis and other diseases (Table-2). 
Histopathological results of forty patients that were examined using light microscope of $\mathrm{H} \& \mathrm{E}$ stained sections. The follicular findings are shown in (Table-3).In addition, the following associated pathological features were also seen such regular and irregular acanthosis in 10(25\%), basal liquefaction in $4(10 \%)$, epidermal atrophy in $3(7.5 \%)$, parakeratosis in $2(5 \%)$, hyperkeratosis in $2(5 \%)$, dyskeratosis in 1
$(2.5 \%)$, churg spire in $1(2.5 \%)$, corpse round and grain in 1(2.5\%,superficial and deep perivascular lymphocyte infiltrations in $8(20 \%)$, pseudoepitheliomatous hyperplasia in $4(10 \%)$, periappeandigeal infiltrations with lymphocytes apart from hair follicle in $4(10 \%)$, solar elastosis in $3(7.5 \%)$,) and absent epidermal melanin in 10 patients
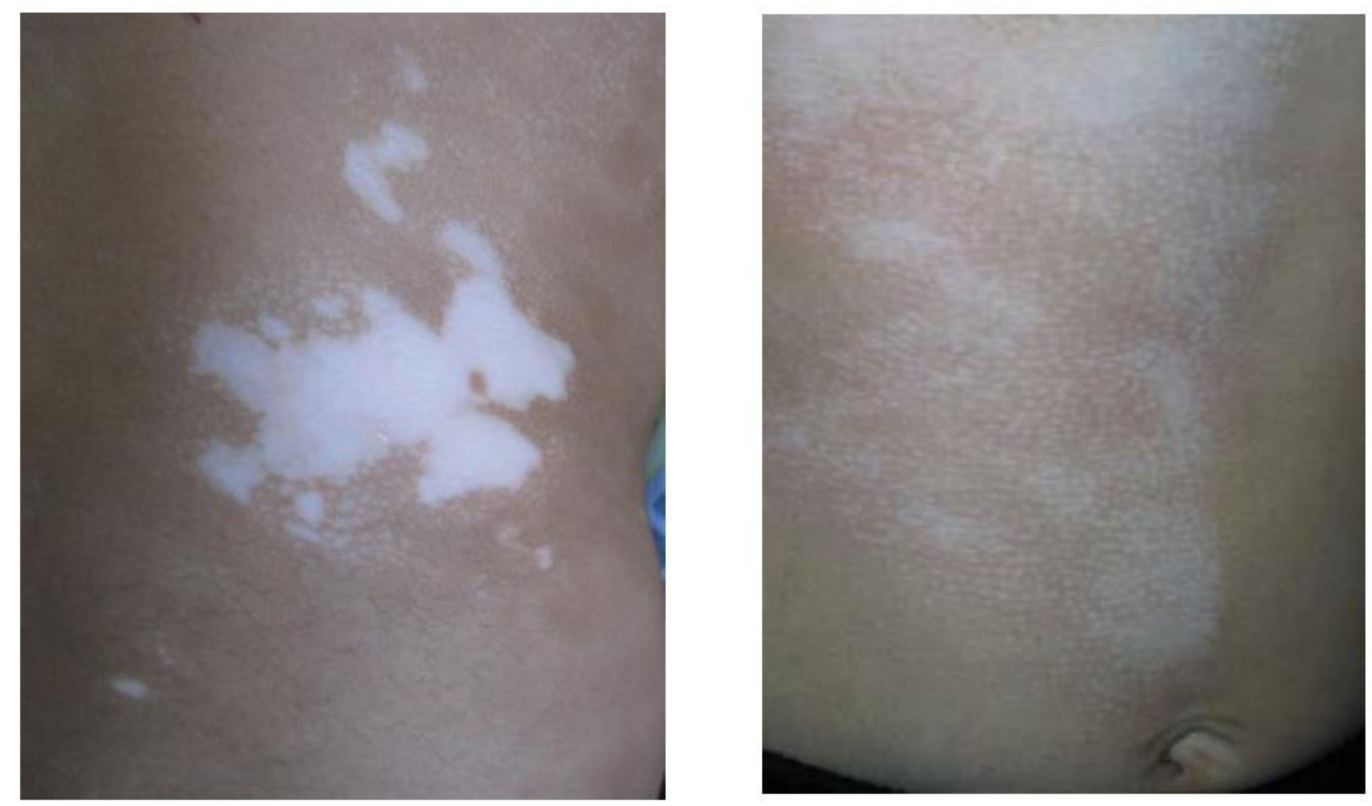

Figure-1: Fourteen years old male with vitiligo showing with follicular lesions.
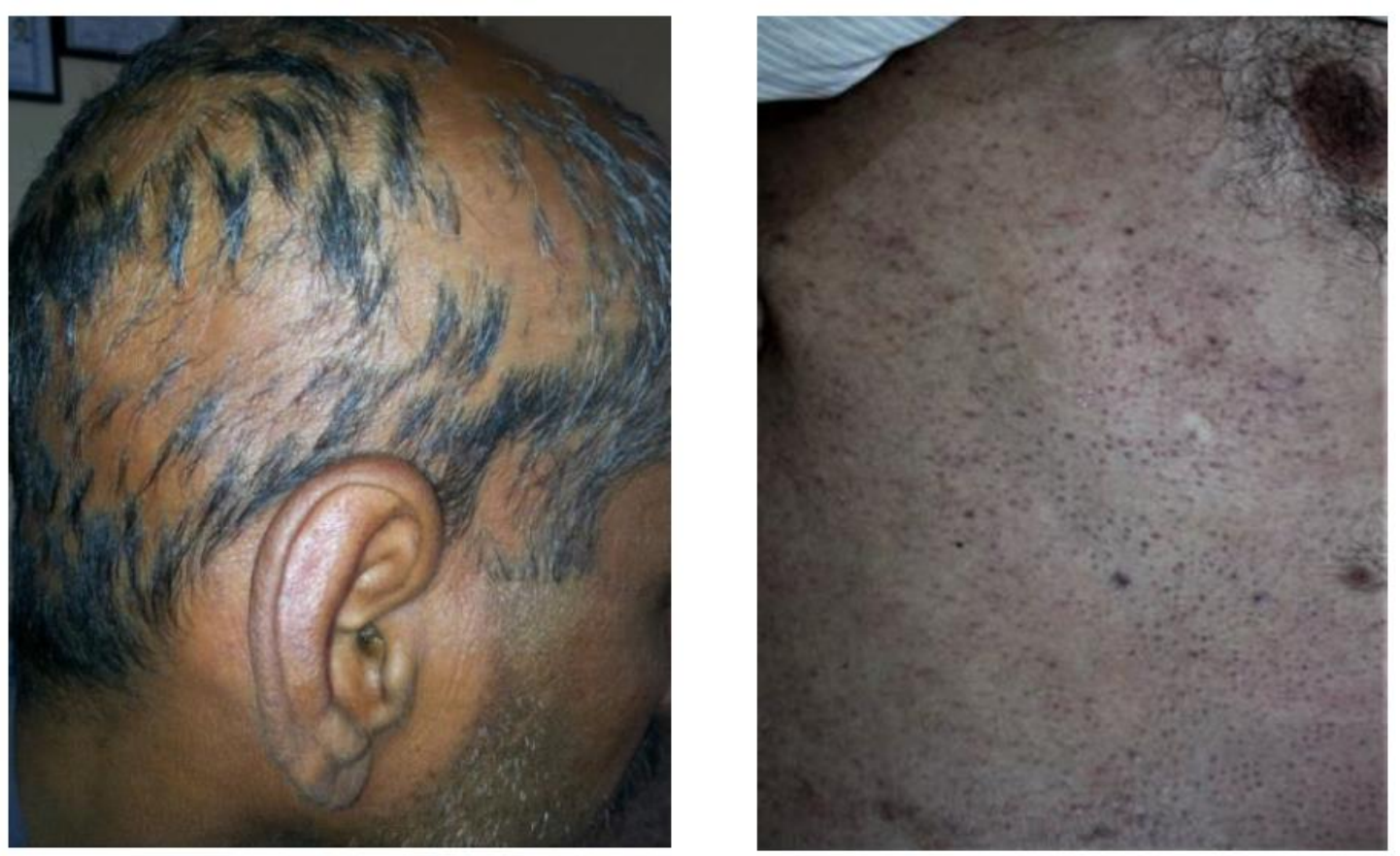

Figure-2: Fifty one years old male with lichen planopilaris showing follicular scarring alopecia with follicular lesions involving the trunk. 
Khalifa E. Sharquie et al., AJCRR, 2020; 4:17
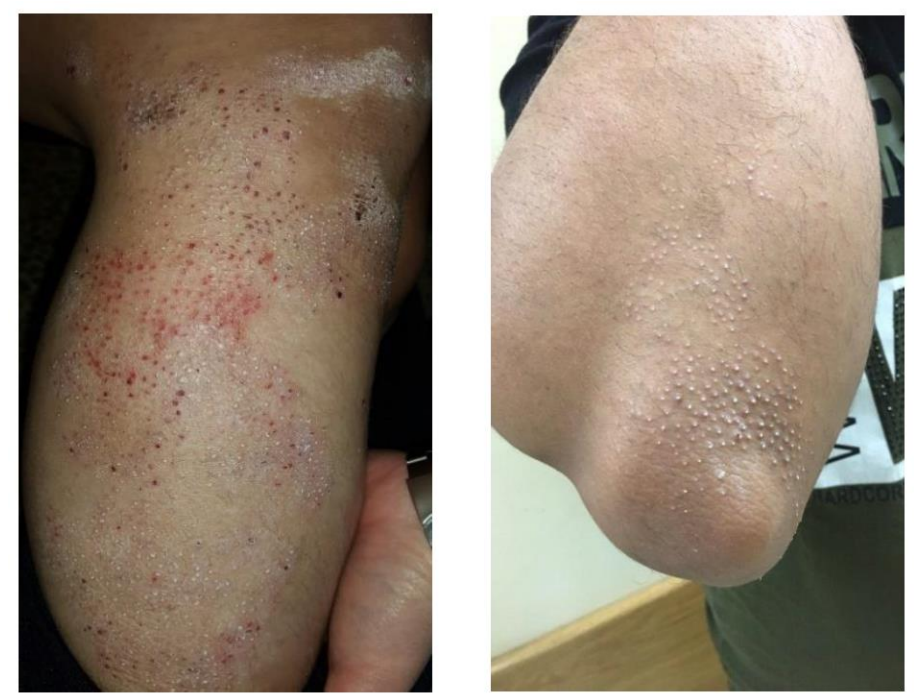

Figure-3: Thirty years old male with psoriasis showing erythematous scaly follicular papules.

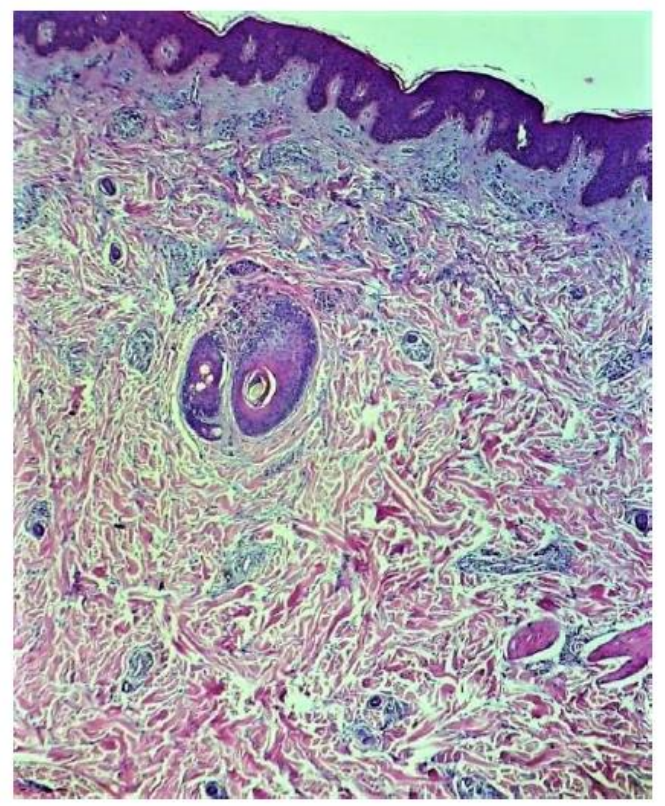

Figure- 4: H\&E strained section of patient with seborrhoeic dermatitis showing perifollicular lymphocytic infiltration.
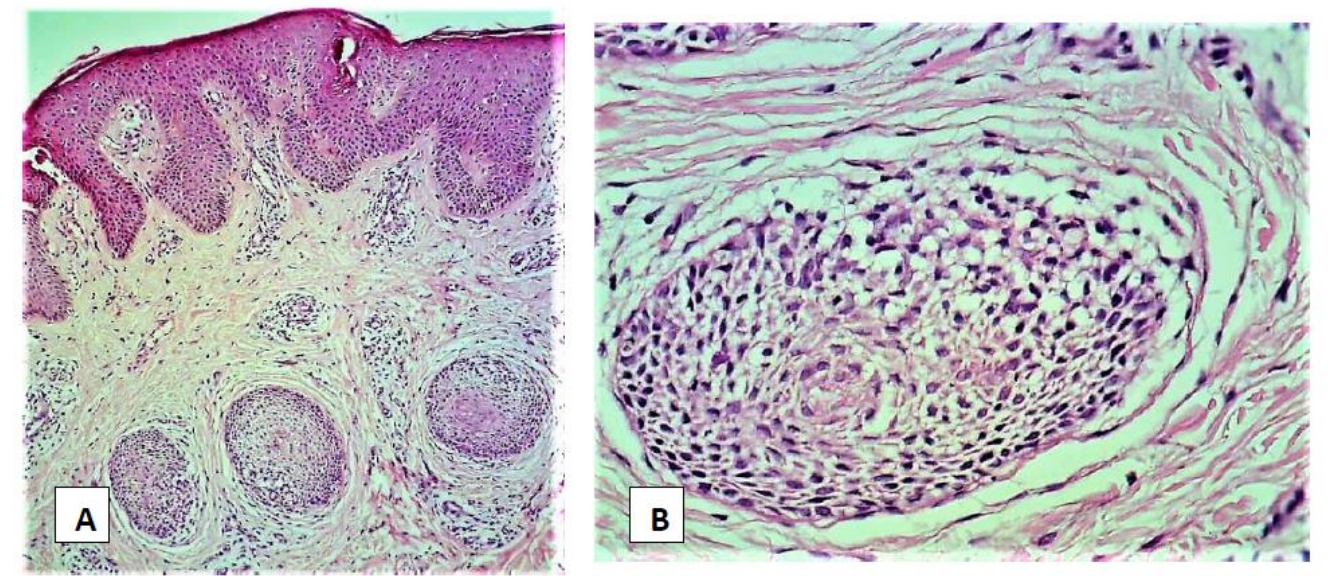

Figure-5: lymphocytic invasion and destruction of the hair follicle. Haematoxylin and Eosin stain. (A- Low mag. 100 and B- high mag. 400). 


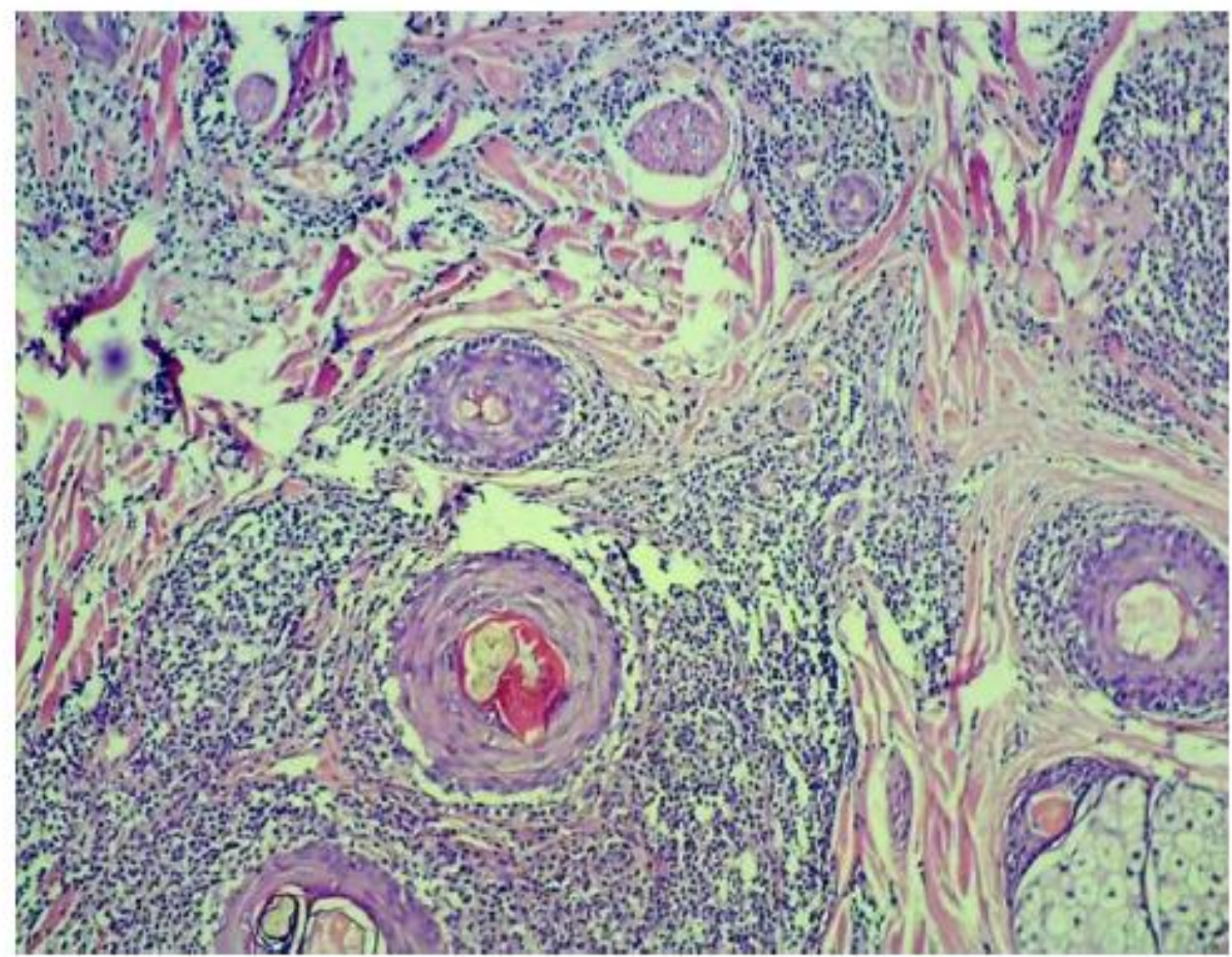

Figure- 6: H\&E stained section of patient with DLE showing follicular plugging, perifollicular lymphocyte infiltration and destruction of the hair follicles. High mag 400.

Table-1: Demographic data of all patients.

\begin{tabular}{|l|l|l|}
\hline Demographic data & Number & Percentage \\
\hline Total number of patients & 60 & $100 \%$ \\
\hline Male patients & 35 & $58.3 \%$ \\
\hline Female patients & 25 & $41.6 \%$ \\
\hline Age range & $1-80$ years & * \\
\hline $\begin{array}{l}\text { Mean } \pm \text { standard deviation of } \\
\text { age }\end{array}$ & $30.2 \pm 16.96$ years & $*$ \\
\hline Number of biopsies & 40 & $66.6 \%$ \\
\hline Symptomatic presentations & 43 & $71.6 \%$ \\
\hline Asymptomatic presentations & 17 & $28.3 \%$ \\
\hline
\end{tabular}


Khalifa E. Sharquie et al., AJCRR, 2020; 4:17

Table-2: Showing clinical diagnosis of the follicular diseases.

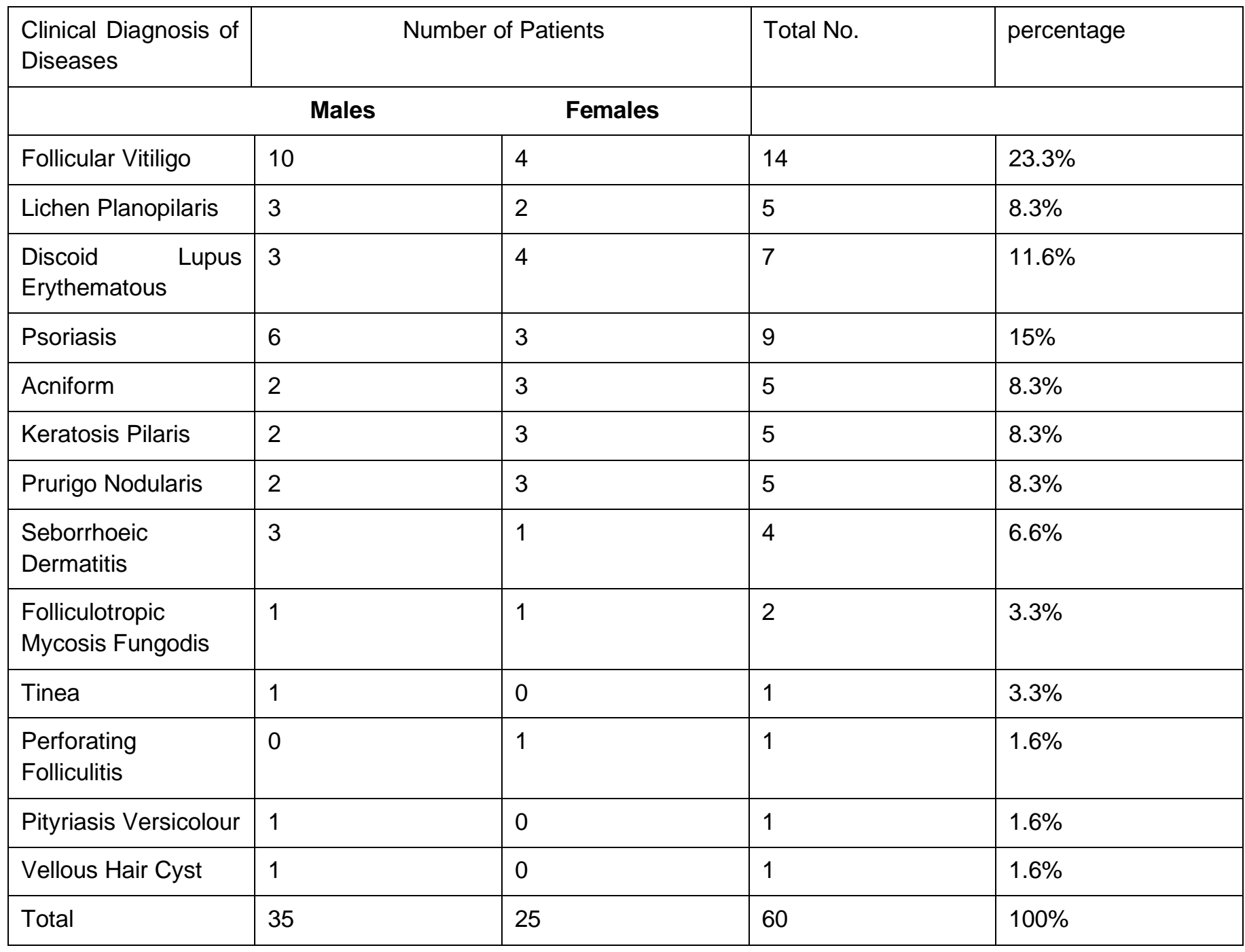

Table -3: Showing the histopathological findings.

\begin{tabular}{|l|l|l|}
\hline Histopathological findings & prevalence & Percentage \\
\hline Perifollicular lymphocytes infiltrations & 40 & $100 \%$ \\
\hline $\begin{array}{l}\text { follicular invasion and degeneration } \\
\text { by lymphocytes }\end{array}$ & 12 & $30 \%$ \\
\hline Follicular plugging & 7 & $17.5 \%$ \\
\hline $\begin{array}{l}\text { Hair follicles damage with fibrous tract } \\
\text { remnant }\end{array}$ & 7 & $17.5 \%$ \\
\hline Perifollicular melanophages & 5 & $12.5 \%$ \\
\hline Absent melanin in the epidermis & 10 & $2.5 \%$ \\
\hline
\end{tabular}

\section{Discussion}

This is the most important study to classify follicular dermatoses in such details all over the world, as it shows full clinical and histopathological evaluation of follicular lesions.
There are many skin diseases with established clinical and histopathological picture of follicular involvement, but still many dermatologists are unaware that the main target in these diseases is the hair follicles. So the aim of present study 
is to collect and categorise of various skin diseases where the hair follicles are primarily involved by the pathological process or these hair follicles are affected during the course of the disease process like in acne, atopic dermatitis, folliculitis, lichen planopilaris and other diseases ${ }^{1,3}$. The hair follicles are important skin appendages, covering almost whole body except few areas like lips, palm and sole, of which its outer root sheath cells in the infundibulum formed by downward extension of epidermis which will be histologically similar to keratinocytes of epidermis and forms a granular layer during keratinazation ${ }^{9}$; this means that the pathological changes that occur in outer root sheath might be identical to the changes that affect the keratinocytes of epidermis. In the isthmus, outer root sheath cells keratinized in a trichilemmal fashion, lacking granular layer and normally express an array of keratins, adhesion molecules, cytokines, and growth factor receptors that are distinct from those expressed by epidermal cells. They migrate out of the follicle and regenerate the epidermis after injury or loss. ${ }^{10}$

Also the immunology of hair is very amazing and complicated as the hair follicle (Figure-7) represents an immune privileged (IP) site like other sites including the anterior chamber of the eye, testis, brain and placenta, which is defined basically as a location in the body where foreign tissue grafts can survive for longer periods of time without immune rejection. This specialized immune environment of IP is required to prevent destructive immune reactions in critical regions. Hair follicle IP has a unique characteristic of recurring in cyclic patterns. Most people think that the IP of the hair follicle is considered to be restricted to the matrix region during the anagen phase. However, evidences have been accumulated to support that the IP of the hair follicle extends to the bulge region and is present at this site during the entire hair cycle. Since the bulge represents the hair follicle stem cell niche, sustained IP in this region may be essential for the survival of the follicle. ${ }^{5}$ Hair follicle IP occurs during anagen ${ }^{11}$ The hair follicle IP is maintained by several factors: Down regulation of MHC class I expression in the proximal ORS and matrix cells, local production of potent immune-suppressants like TGF- $\beta 1$, IL- 10 and $\alpha$ $\mathrm{MSH}$, functional deterioration of antigen presenting cells, absence of lymphatic, establishment of extracellular matrix barriers to hinder immune cell trafficking, expression of non-classical MHC class 1, and Expression of Fas ligand. ${ }^{12}$ The hair follicles have a complex immunologic privileges, with immunologically "privileged" matrix cells at its base, and a complement of perifollicular macrophages, mast cells, and other immunocytes that act as the effector arm of the immune system. ${ }^{10}$ However; the hair follicles are suspended inside the dermis; hence; has more extensive surface area that exposed to any surrounding pathological process. In addition, the hair follicle has its distinct blood supply. This does indicate that there will be more intense inflammatory reaction in the hair follicle in comparison with the neighbouring basal layer of epidermis where there is only one surface of basal keratinocytes is exposed to dermis. Also we can think that the antigenic stimulation still could be more intense in hair follicles than on epidermis; as the density of various antigens or receptors could be more intense in the keratinocytes of outer root sheath than those of epidermis. Accordingly the immunological and pathological reaction should be more florid. Hence we expect that all inflammatory skin diseases might start initially at hair follicles and will be more intense than the surrounding epidermal cells. This inflammatory will be presented as a minute macules or papules that coalesce together to form plaques or patches as seen in vitligo,psoriasis, seborrhoic dermatitis and many other diseases . 6- 8 The immunological and inflammatory damage might involve the bulb of the hair as in alopecia areata and grey hair vitiligo or involve the shaft of the hair as in LPP and DLE. Similarly, when there is hyperkeratosis of skin, it will be more obvious at the orifices of the hair follicles than the surrounding epidermis as seen in many 
cases of keratosis pillars. ${ }^{2}$ Although the disease start as follicular lesions but this change could be microscopic and not seen by the naked eye or clinically obvious and easily seen. So the question that should be rose again why the follicular lesions could not be observed all the time clinically or histopathologically? The following points will give the final answers:

1- The lesion could be so minute that could not be easily seen.

2- The pathological changes could be so minimal that could not be observed on histopathological section.

3- The hair follicle involvement could be missed during sectioning of the biopsy.
4- The follicular lesion could be transient and then spread to actual epidermis.

There are many reports in the medical literature emphasizing the importance of the hair follicle in the disease process but are rarely reported in one solid study, in contrast to the present work where we had definitely confirmed the importance of the hair follicles in etiopathogenesis of the diseases. So the present work had confirmed this observation.

Finally somebody will ask what about in areas where there are no hair follicles like palms and lips? Here in these areas the orifice of sweat glands will represent the orifice of hair follicles. 13

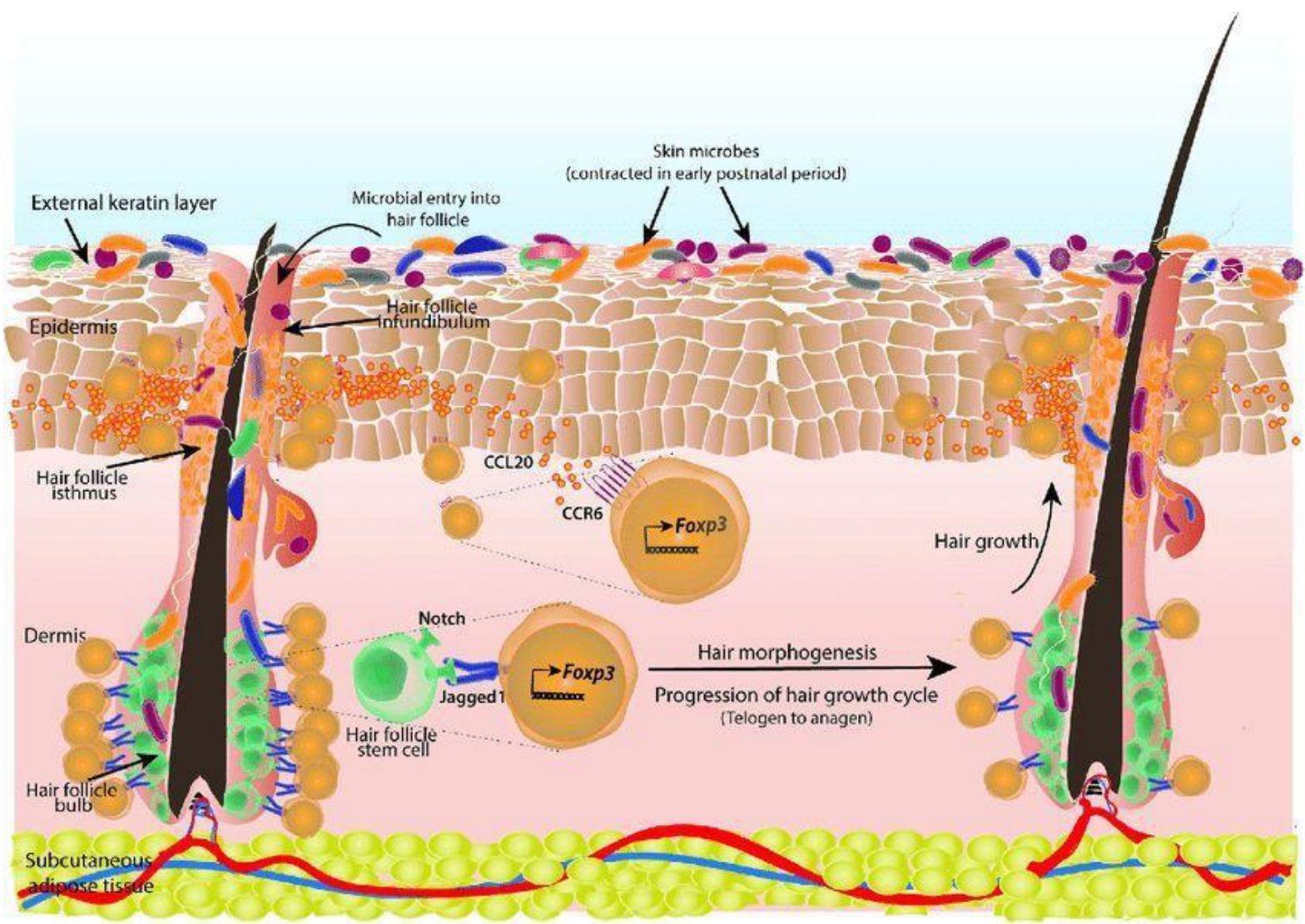

Figure -7: Showing the immune privilege of Hair follicles ${ }^{14}$

\section{Conclusion}

The hair follicles have pathological and immunological privileges as they are suspended anatomical structures inside the dermis. The results of the present work concluded that almost all skin diseases start initially in the hair follicle as minute macules, papules and then spread to involve the adjacent epidermis and then coalesce together to form large lesions. Also, these immunological and pathological events that involve the hair follicles could be microscopically and transient clinically and histopathologically that easily missed.

\section{References}

1. Sharquie KE, Noaimi AA, Mijthab ZM. Chronic scalp folliculitis versus acne vulgaris 
(observational case series study). J Clin Exp Dermatol Res. 2012; 3(153):2.

2. Thomas M, Khopkar US. Keratosis pilaris revisited: is it more than just a follicular keratosis? Int J Trichol 2012; 4:255-8.

3. Sharquie KE, Noaimi AA, Hameed AF. Lichen Planopilaris is a Common Scarring Alopecia among Iraqi Population. Journal of Cosmetics, Dermatological Sciences and Applications. 2013 Aug 16; 3(3):35-9.

4. Messenger AG, de Berker DA, \& Sinclair RD. Disorders of hair in: Burns T, Breathnach S, Cox N, Griffiths C. Rook's Text Book of Dermatology. 8th ed. UK: Wiley-Blackwell, 2010; 4, 66: 1-11.

5. Erdoğan B. Anatomy and Physiology of Hair. In Hair and Scalp Disorders 2017 May 3. (2):21.

6. Thomas LJ, Dadzie OE, Francis N, Morar N. Follicular Psoriasis-A Forgotten Entity?. Open Dermatology Journal. 2010/4:95-6.

7. Berth-Jones J. Eczema, lichenification, prurigo and erythroderma in: Burns T, Breathnach S, Cox N, Griffiths C. Rook's Text Book of Dermatology. 8th ed. UK: Wiley-Blackwell, 2010; Mar 22/1:1-51.
8. Sharquie KE, Noaimi AA. Follicular vitiligo: the present clinical status. Our Dermatology Online. 2016 Apr 1; 7(2):176.

9. Cotsarelis G, Botchkarev V. Biology of hair follicles. In: Goldsmith LA, Kataz SI, Gilchrest BA, Paller SA, Leffell DJ, Wolf K (eds). Fitzpatrick's Dermatology in General Medicine, 8th ed. New York, McGrow Hill Company, 2012; 1, 15, 86: 960-8.

10. Paus $R$, Cotsarelis $G$. The biology of hair follicles. New England journal of medicine. 1999 Aug 12; 341(7):491-7.

11. Christoph T, Müller-Röver S, Audring $H$. The human hair follicle immune system: cellular composition and immune privilege. . British Journal of Dermatology, 2000/142:862-73.

12. Paus $R$, Ito $N$, Takigawa $M$. The hair follicle and immune privilege. J Investig Dermatol Symp Proc 2003/8:188-94.

13. Groscurth P. Anatomy of sweat glands. InHyperhidrosis and botulinum toxin in dermatology 2002, 30, 1-9. Karger Publishers.

14. https://www.researchgate.net/figure/Cutaneousregulatory-T-cells-Tregs-facilitate-hairmorphogenesis-and-establish_fig3_324750840

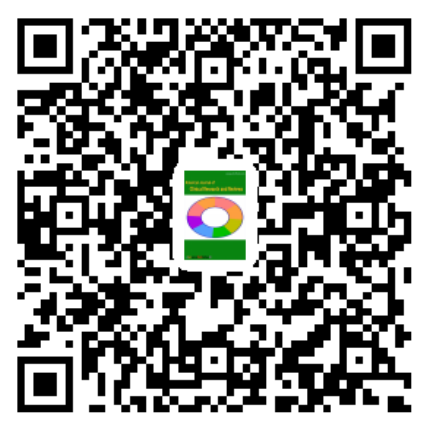

